Int. J. Dev. Biol. 55: 563-567

doi: $10.1387 / \mathrm{ijdb} .103231 \mathrm{rh}$

\title{
Escape mechanisms after antiangiogenic treatment, or why are the tumors growing again?
}

\author{
RUSLAN HLUSHCHUK ${ }^{1}$, ANDREW N. MAKANYA ${ }^{1,2}$ and VALENTIN DJONOV*,1,3 \\ ${ }^{1}$ Department of Medicine, University of Fribourg, Fribourg, Switzerland, ${ }^{2}$ Department of Vet Anatomy \& Physiology, \\ University of Nairobi, Nairobi, Kenya and ${ }^{3}$ Institute of Anatomy, University of Bern, Bern, Switzerland
}

\begin{abstract}
Inhibitors of angiogenesis and radiation induce compensatory changes in the tumor vasculature both during and after cessation of treatment. In numerous preclinical studies, angiogenesis inhibitors were shown to be efficient in the treatment of many pathological conditions, including solid cancers. In most clinical trials, however, this approach turned out to have no significant effect, especially if applied as monotherapy. Recovery of tumors after therapy is a major problem in the management of cancer patients. The mechanisms underlying tumor recovery (or therapy resistance) have not yet been explicitly elucidated. This review deals with the transient switch from sprouting to intussusceptive angiogenesis, which may be an adaptive response of tumor vasculature to cancer therapy that allows the vasculature to maintain its functional properties. Potential candidates for molecular targeting of this angioadaptive mechanism are yet to be elucidated in order to improve the currently poor efficacy of contemporary antiangiogenic therapies.
\end{abstract}

KEY WORDS: antiangiogenic therapy, anticancer therapy, protein tyrosine kinase inhibitor, tumor recovery, intussusceptive angiogenesis, escape mechanism

\section{Tumor angiogenesis}

Angiogenesis, the process of formation of new blood vessels from pre-existing ones, plays an essential role in the tumour growth and progression. New vascular segments are needed to supply the increasing tumour mass with oxygen and nutrients. Hence, the crucial step for tumour growth above the size of 1-2 $\mathrm{mm}^{3}$ is the "angiogenic switch", when the tumour gains capability to produce angiogenic factors and induce angiogenesis (Hanahan and Folkman, 1996).

\section{Modes of angiogenesis}

Two major modes of angiogenesis have been described, namely sprouting and intussusception (often also called "non-sprouting" or splitting) (Burri et al., 2004). Sprouting angiogenesis was described more than 150 years ago and has been in the focus of research groups for many decades. This mode of angiogenesis occurs by outgrowth of abluminal sprouts which subsequently merge with existing capillaries. Degradation of endothelial basement membranes (obviously associated with increased vessel permeability) as well as proliferation of endothelial cells are essential for this process (van Hinsbergh and Koolwijk, 2008). This mode of angiogenesis is the more widely studied and many of the molecular and cellular mechanisms involved have already been elucidated (see reviews (Carmeliet et al., 2009; van Hinsbergh and Koolwijk, 2008).

In contrast, intussusceptive angiogenesis (IA) is achieved by intraluminal growth. So far undetermined factors trigger the formation of endothelial cell processes on the opposing sides of the vascular lumen. A transluminal pillar, the hallmark of intussusceptive angiogenesis, is formed when these opposing endothelial protrusions extend and finally make contact (Burri et al., 2004). The steps in the formation of a transluminar pillar, the basic event in IA, are presented in Fig. 1.

Intussusceptive angiogenesis may have different outcomes depending on location of the pillars: intussusceptive microvascular growth (IMG), refers to the expansion of the capillary bed; intussusceptive arborization (IAR) describes the formation of the typical vascular tree while intussusceptive branching remodeling (IBR) denotes vascular remodeling and adaptation to suit local perfusion requirements and includes intussusceptive vascular pruning (IVP)

Abbreviations used in this paper: $\mathrm{bFGF}$, basic fibroblast growth factor; IA, intussusceptive angiogenesis; IAR, intussusceptive arborization; IBR, intussusceptive branching remodeling; IMG, intussusceptive microvascular growth; IVP, intussusceptive vascular pruning; VEGF, vascular endothelial growth factor.

\footnotetext{
*Address correspondence to: Valentin Djonov. Institute of Anatomy, University of Bern, Baltzerstrasse 2, 3000 Bern-9, Switzerland.

Fax: +4131631 3807. e-mail: valentin.djonov@ana.unibe.ch
}

Final, author-corrected PDF published online: 27 July 2011.

ISSN: Online 1696-3547, Print 0214-6282

(C) 2011 UBC Press

Printed in Spain 

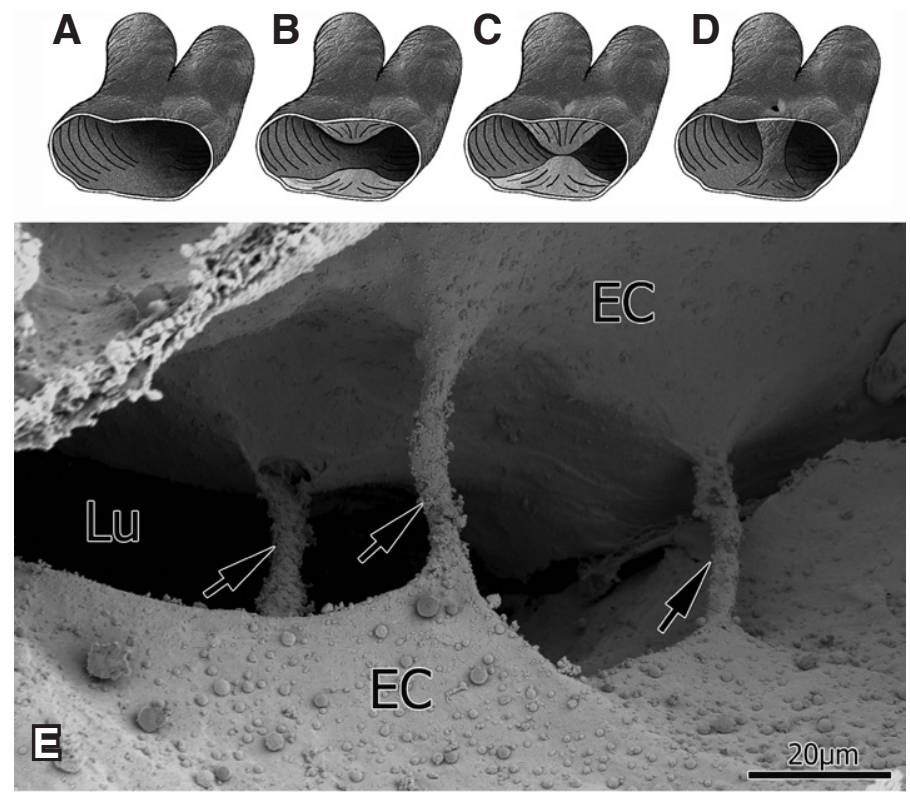

Fig. 1. Demonstration of the mechanisms involved in pillar formation. (A-D) Three-dimensional schema illustrating the steps in the formation of transluminal pillars during intussusceptive angiogenesis. The process begins with the protrusion of portions of the walls from opposite sides into the vessel lumen (A,B). After contact has been established and "corroborated" (C), the endothelial bilayer becomes perforated centrally and a transluminal pillar is formed (D). The scanning electron microphotograph of critical-point-dried and sputtered with gold sample is presented in $\mathbf{E}$. It displays the intraluminal view of the sinusoidal vessel typical for intussusception with many transluminal pillars (arrows). EC, endothelial cell; Lu, lumen. (A-D) are adapted from (Djonov et al., 2003); (E) from Makanya et al., unpublished data.

(see reviews (Burri et al., 2004; Makanya et al., 2009)). Intussusceptive angiogenesis permits a rapid and enormous increase in the number of microvessels (IMG) without concomitant proliferation of endothelial cells or increase in the vessel permeability as may occur due to degradation of the basement membrane s (Ausprunk et al., 1974; Burri and Djonov, 2002; Djonov et al., 2003).

Intussusception is complementary to sprouting during organogenesis. According to our own data the vasculature of different organs undergoes two main phases of development: an early sprouting phase is followed by an intussusceptive phase, during which capillary sprouting is superseded by transluminal pillar formation. Intussusception results in further expansion of the capillary plexus and formation of an organ-specific vascular tree, as well as its dynamic adaptation, including intussusceptive vascular pruning (see review by Makanya et al., 2009). It is noteworthy that during the sprouting phase VEGF expression level is high and it is considerably decreased during the intussusceptive phase, while the level of basic fibroblast growth factor (bFGF) is increasing with advance from former to the latter phase (Makanya et al., unpublished data). This finding indicates possible involvement of bFGF in intussusception. In contrast to sprouting, the signaling pathways and molecular mechanisms involved in the process of intussusception are largely unknown.

Other less well characterized forms of angiogenesis are wrapping and hollowing, glomeruloid angiogenesis, co-option of pre-existing vessels or vasculogenic mimicry (Dome et al., 2007).

\section{Rationale behind antiangiogenic cancer therapy}

Lack of adequate vasculature restricts tumor volume progression that should result from continuous cell proliferation, the hallmark of cancer pathogenesis (Hanahan and Folkman, 1996). Therefore, the control over the tumor vasculature (tumour-induced angiogenesis) should provide the control over the tumor growth.

The idea of cancer treatment by inhibiting pro-angiogenic pathways was introduced by Folkman in 1971 and can be considered an official "start" of the era of antiangiogenic research (Folkman, 1971). It was expected that the first successful antiangiogenic cancer therapy would be accomplished within the subsequent few years. It took much longer than that: about 4 decades passed before the first antiangiogenic drug, bevacizumab, was approved for cancer treatment in clinical practice (Kramer and Lipp, 2007). Angiogenesis inhibitors were shown to be efficient in the treatment of many pathological conditions, including solid cancers. There are over two thousand clinical trials based on angiogenesis inhibitors (http://clinicaltrials.gov/ct2/results?intr=\%22Angiogenesis+Inhibito rs \%22\&pg=2\&show_flds $=Y$ ), but in most of the completed studies they turned out to have no significant effect, especially if applied as monotherapy (Soltau and Drevs, 2009).

\section{Anti-VEGF (anti-sprouting) approach and its efficacy}

Most of the antiangiogenic drugs under investigation target the signaling pathway of vascular endothelial growth factor (VEGF), also known as vascular permeability factor, the most potent angiogenic factor described this far. VEGF augments proliferation and migration of endothelial cells (the hallmarks of sprouting), increases the permeability of vessels (Bates et al., 2002) and causes vasodilatation (Ku et al., 1993). Moreover, it is also known as a survival factor for endothelial cells (Gerber et al., 1998) and a negative regulator of pericyte function and vessel maturation (Greenberg et al., 2008). Therefore, inhibition of VEGF signaling should inhibit proliferation and migration of endothelial cells, decrease permeability of the vessels, prevent vasodilatation (=vasoconstriction?), and result in increased apoptosis of the endothelial cells as well as pericyte activation and vessel maturation. The single effects mentioned above can be and are actually achieved to some great extent by existing anti-VEGF drugs (at least transiently), the ultimate goal of antiangiogenic cancer treatment - the long-term control of tumour growth, however, is unfortunately out of easy reach at the moment (Broxterman et al., 2003; Ellis and Hicklin, 2008; Miller et al., 2003; Miller et al., 2005).

In this review we would like to introduce a hypothesis that could probably better explain the angioadaptive mechanisms of tumour vasculature in response to antiangiogenic treatment, as well as some of the cases of the resistance to antiangiogenic (anti-VEGF) treatment.

\section{Hypothesis of transient vascular normalization}

Tumour recovery after therapy is a major problem in the management of cancer patients. The survival benefits of antiangiogenic drugs have thus far been rather disappointing, hence stimulating interest in developing more effective ways to combine antiangiogenic drugs with established chemotherapeutic approaches (Carmeliet et al., 2009; Ma and Waxman, 2008). This situation has also motivated 
many research groups to investigate the mechanisms underlying such a low efficacy of anti-angiogenic treatment, to understand the compensatory changes in tumour vasculature induced by inhibitors of angiogenesis. Few hypotheses and molecular mechanisms that could explain the resistance to antiangiogenic treatment in particular cases were suggested (e.g., see reviews Bergers and Hanahan, 2008; Ellis and Hicklin, 2008). The 'normalization hypothesis' introduced by Rakesh Jain is the most popular nowadays and postulates that antiangiogenic treatment leads to transient normalization of the vasculature, what improves the perfusion of the tumour tissue. This transient normalization should therefore be beneficial for the delivery of co-applied cytostatic therapy (Jain 2005; Jain et al., 2006).

According to Jain, the abnormal tumour vasculature becomes "more normal" during its treatment with antiangiogenic drugs (Jain 2005). The major observations (events/phenomena) that are included in the "normalization" term (phenomenon) are the following:

- Decrease in intratumoural microvascular density by pruning

of leaky vessels

- Increase in pericyte coverage with:

- decreased vascular permeability and

- improved perfusion (what subsequently leads to improved

drug penetration)

- Second wave of angiogenesis

This hypothesis is explained using the "balance of pro- and anti-angiogenic factors": when the proangiogenic factors prevail angiogenesis is stimulated and the vessels are "abnormal". Application of antiangiogenic drugs brings back the balance of pro- and antiangiogenic factors. This restored balance results in reduction of "abnormality" of tumour vessels. The explanation is easily (intuitively?) understood and convincing. The number of contemporary investigators that support this hypothesis (including authors of the present review) is steadily increasing. The missing component is a more detailed investigation of the morphogenic mechanism(s).

\section{Angiogenic switch from sprouting to intussusception}

The transient normalization of tumour vasculature (including all the aforementioned observed phenomena) may be explained on morphogenic level by the angiogenic switch from sprouting to intussusception. This switch is an angioadaptive mechanism of tumor vasculature to the antiangiogenic factors (Hlushchuk et al., 2008). Occurrence of intussusception has been demonstrated in various tumours (Hillen and Griffioen, 2007) and its activation is evident during blockage of sprouting by anti-VEGF therapy (Hlushchuk et al., 2008). In our recent study we have shown that the mode of vasculature growth of xenograft tumours treated with PTK/ZK (VEGFR inhibitor) undergoes shifting from sprouting to intussusception after antiangiogenic treatment or radiotherapy. Activation of intussusception, specifically intussusceptive pruning, leads to decrease in the intratumoural microvascular density in treated tumours but without decrease in vascular exchange surface area and stimulates vessel wall maturation (i.e., decreased vascular permeability and improved perfusion). Moreover, the switch to intussusceptive mode of angiogenesis improves the perfusion of the tumour mass as has been shown by the decrease of in hypoxia of the tumour mass after the antiangiogenic treatment or radiotherapy (Hlushchuk et al., 2008). Improvement in oxygen supply represents better tumour perfusion and therefore should also mean improved drug penetration into the tumour tissue as observed during the "transient vascular normalization" (Jain 2008). All the features of the angiogenic switch from sprouting to intussusception mentioned above match the phenomena known together as "transient vascular normalization" (Jain, 2005).

Notably, intussusceptive angiogenesis subsequent to treatment with PTK/ZK has already been documented (albeit unbeknown to the authors) in other tumor models, such as in the murine renal cell carcinoma study (Drevs et al., 2002). In the latter study PTK/ ZK elicited changes in the vasculature that were typical of intus-

A
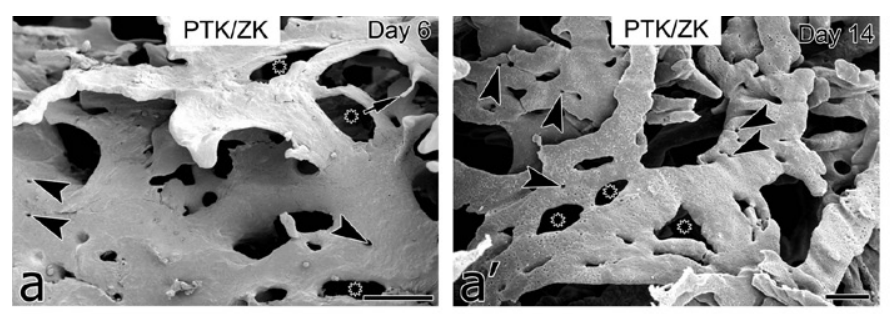

B

NO TREATMENT

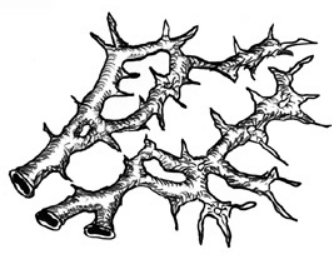

Sprouting

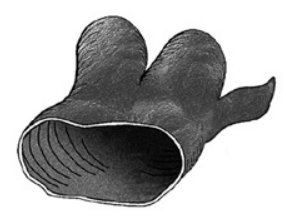

RECOVERY

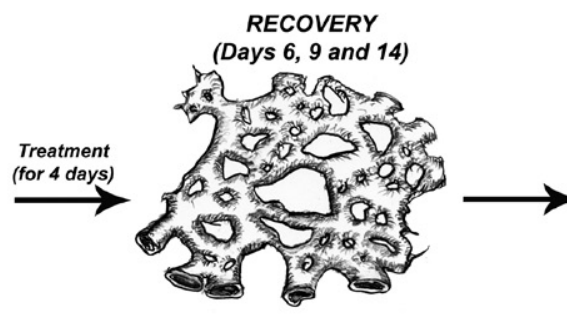

Intussusceptive phase

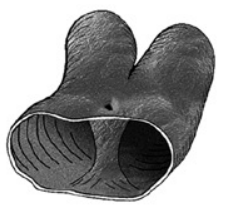

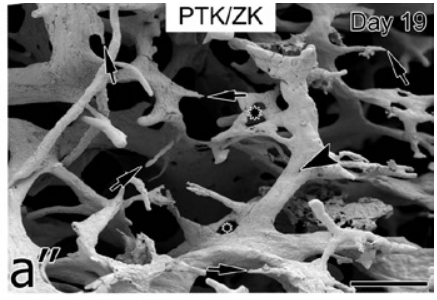

END OF RECOVERY

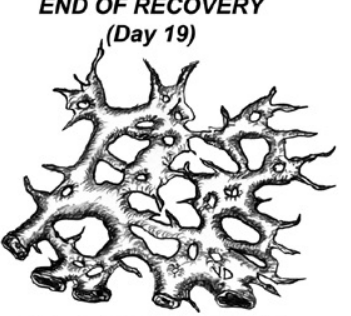

Second wave of sprouting

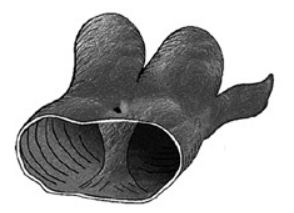

Fig. 2. Time-course of the angiogenic switch during tumor recovery. (A) The scanning electron micrographs representing the vascular pattern of the PTK/ZK treated tumors on day 6 (a), 14 (a') and 19 $\left(a^{\prime \prime}\right)$. On day 6 and 14 pillars (arrowheads) and meshes (asterisks) dominate. In contrast to day 6 and 14, on day 19 (a") the sprouts (arrows) prevail in the vascular pattern of the PTK/ZK-treated tumors with some pillars (arrowhead) or meshes (asterisks) present. The scale bar in a-a" is $100 \mu \mathrm{m}$. The scheme in $\mathbf{( B )}$ represents the above-described changes: in non-treated tumors the dominating mode of angiogenesis is sprouting. After the short-term therapy the intussusception prevails: so called, intussusceptive phase of recovery (days 6, 9 and 14). On day 19 the second wave of intussusception is there what is characterized by numerous sprouts along with pillars or meshes. Adapted from (Hlushchuk et al., 2008). 
susception. The vessel density appeared to be decreased by $48 \%$ while the systolic blood-flow velocity in the renal artery remained unchanged and the vessel permeability decreased by $50 \%$ (Drevs et al., 2002). Furthermore, although the vascular casts presented clearly revealed the presence of transluminal pillars, the authors did not recognize the phenomenon of intussusceptive angiogenesis. Similar changes in vasculature have been observed in murine orthotopic B16/BL6 melanoma tumor model after treatment with either PTK/ZK (Rudin et al., 2005) or other tyrosine kinase inhibitors (Nakamura et al., 2004; Nakamura et al., 2006; Ruggeri et al., 2003). In each instance, the depicted changes in the vasculature corresponded to those associated with intussusception. Intussusceptive angiogenesis might represent a general process as part of a tumor-protective adaptive response (Hlushchuk et al., 2008).

After some time (in our study it was 2 weeks after cessation of therapy) the tumour vasculature returns back to the sprouting mode of angiogenesis, what corresponds to the relapse of the tumour (the subsequent wave of sprouting angiogenesis coincides with tumour growth). This observation is consistent with the findings of Hanahan and his collaborators (Casanovas et al., 2005) in which the "second wave of angiogenesis" occurred after 4 weeks of antiangiogenic treatment. According to Rakesh Jain, the second wave of angiogenesis may signalize the closure of 'transient vascular normalization' window (Jain 2008). Here we would like to emphasize that it is the second wave of sprouting (!) angiogenesis: it correlates perfectly with the findings of our study that after the transient switch to intussusception the second wave of sprouting angiogenesis took place (Fig. 2). Putatively the tumour vasculature that undergoes such an angiogenic switch is much more resistant to further antiangiogenic treatment or radiotherapy: the vessels become more mature and thus more rigid and less vulnerable to VEGF inhibition (Helfrich et al., 2010; Hlushchuk et al., 2007; Sennino et al., 2009).

\section{bFGF as potential key molecule}

The molecular players involved in intussusception are largely unknown. At present, probably the most prominent candidate involved in intussusception is basic fibroblast growth factor (bFGF). It was reported that bFGF may compensate for the loss of VEGF after the tumors have reached a certain size (Yoshiji et al., 1997). Its plasma levels appeared to be increased and correlated with vessel size in patients who experienced tumor progression while on AZD2171 (cediranib), an oral VEGFR inhibitor (Batchelor et al., 2007), confirming the aforementioned findings of Hanahan and coworkers in the mouse model of oncogene-induced insulinomas (Casanovas et al., 2005). The findings from the aforementioned preclinical and clinical studies can also be interpreted to imply that bFGF upregulation coincides with tumor relapse (or precedes it?). It could be one of the key molecules responsible for activation of intussusception when sprouting is blocked by anti-VEGF drugs. That would correlate with over-expression of bFGF during activation of intussusceptive remodeling and pruning as found in the developing chick embryonic kidney (Makanya et al., unpublished data)).

Another strong point of bFGF being involved in the angiogenic switch to intussusception is that targeting bFGF may provide synergistic effects in the treatment of angiogenesis-related disorders, including cancer (see review Alessi et al., 2009), as well as the suggestion to use it as a biomarker for efficacy of (resistance to) antiangiogenic therapy as well as potential target for extending the normalization window (Batchelor et al., 2007). Probably, it may serve as a marker of intussusception.

\section{Summary}

In this review we hypothesise that the angiogenic switch from sprouting to intussusceptive mode of angiogenesis may be the morphogenic mechanism underlying the widely accepted hypothesis of transient vascular normalization during antiangiogenic (antisprouting) treatment. All the described changes in the "normalized" vasculature of treated tumours are typical of intussusceptive remodelling, including intussusceptive vascular pruning. Although the molecular mechanisms of intussusception are largely unknown we suggest taking into consideration bFGF as potential candidate for the key role in intussusception.

Plausibly, manipulation of this escape mechanism (switch to intussusceptive mode of angiogenesis) would lead to new levels of antiangiogenic treatment. Thus, potential candidates for molecular targeting of this angioadaptive mechanism need to be investigated in order to improve the current poor efficacy of contemporary antiangiogenic therapies.

\section{References}

ALESSI P, LEALI D, CAMOZZI M, CANTELMO A, ALBINI A, PRESTA M (2009). Anti-FGF2 approaches as a strategy to compensate resistance to anti-VEGF therapy: long-pentraxin 3 as a novel antiangiogenic FGF2-antagonist. Eur Cytokine Netw 20: 225-234.

AUSPRUNK DH, KNIGHTON DR, FOLKMAN J (1974). Differentiation of vascular endothelium in the chick chorioallantois: a structural and autoradiographic study. Dev Biol 38: 237-248.

BATCHELOR TT, SORENSEN AG, DI TE, ZHANG WT, DUDA DG, COHEN KS, KOZAK KR, CAHILL DP, CHEN PJ, ZHU M, ANCUKIEWICZ M, MRUGALA MM, PLOTKIN S, DRAPPATZ J, LOUIS DN, IVY P, SCADDEN DT, BENNER T, LOEFFLER JS, WEN PY, JAIN RK (2007). AZD2171, a pan-VEGF receptor tyrosine kinase inhibitor, normalizes tumor vasculature and alleviates edema in glioblastoma patients. Cancer Cell 11: 83-95.

BATES DO, HILLMAN NJ, WILLIAMS B, NEAL CR, POCOCK TM (2002). Regulation of microvascular permeability by vascular endothelial growth factors. $J$ Anat 200: 581-597.

BERGERS G, HANAHAN D (2008). Modes of resistance to anti-angiogenic therapy Nat Rev Cancer 8: 592-603.

BROXTERMAN HJ, LANKELMA J, HOEKMAN K (2003). Resistance to cytotoxic and anti-angiogenic anticancer agents: similarities and differences. Drug Resist Updat 6: 111-127.

BURRI PH, DJONOV V (2002). Intussusceptive angiogenesis--the alternative to capillary sprouting. Mol Aspects Med 23: S1-27.

BURRI PH, HLUSHCHUK R, DJONOV V (2004). Intussusceptive angiogenesis: its emergence, its characteristics, and its significance. Dev Dyn 231: 474-488.

CARMELIETP, DE SF, LOGESS, MAZZONEM (2009). Branching morphogenesis and antiangiogenesis candidates: tip cells lead the way. Nat Rev Clin Oncol6:315-326.

CASANOVAS O, HICKLIN DJ, BERGERS G, HANAHAN D (2005). Drug resistance by evasion of antiangiogenic targeting of VEGF signaling in late-stage pancreatic islet tumors. Cancer Cell 8: 299-309.

DJONOV V, BAUM O, BURRI PH (2003). Vascular remodeling by intussusceptive angiogenesis. Cell Tissue Res 314: 107-117.

DOME B, HENDRIX MJ, PAKU S, TOVARI J, TIMAR J (2007). Alternative vascularization mechanisms in cancer: Pathology and therapeutic implications. $A m \mathrm{~J}$ Pathol 170: 1-15.

DREVS J, MULLER-DRIVER R, WITTIG C, FUXIUS S, ESSER N, HUGENSCHMIDT H, KONERDING MA, ALLEGRINI PR, WOOD J, HENNIG J, UNGER C, MARME D (2002). PTK787/ZK 222584, a specific vascular endothelial growth 
factor-receptor tyrosine kinase inhibitor, affects the anatomy of the tumor vascular bed and the functional vascular properties as detected by dynamic enhanced magnetic resonance imaging. Cancer Res 62: 4015-4022.

ELLIS LM, HICKLIN DJ (2008). Pathways mediating resistance to vascular endothelial growth factor-targeted therapy. Clin Cancer Res 14: 6371-6375.

FOLKMAN J (1971). Tumor angiogenesis: therapeutic implications. N Engl J Med 285: 1182-1186.

GERBER HP, DIXIT V, FERRARAN (1998). Vascular endothelial growth factor induces expression of the antiapoptotic proteins $\mathrm{Bcl}-2$ and $\mathrm{A} 1$ in vascular endothelial cells. J Biol Chem 273: 13313-13316.

GREENBERG JI, SHIELDS DJ, BARILLAS SG, ACEVEDO LM, MURPHYE, HUANG J, SCHEPPKE L, STOCKMANN C, JOHNSON RS, ANGLE N, CHERESH DA (2008). A role for VEGF as a negative regulator of pericyte function and vessel maturation. Nature 456: 809-813.

HANAHAN D, FOLKMAN J (1996). Patterns and emerging mechanisms of the angiogenic switch during tumorigenesis. Cell 86: 353-364.

HELFRICH I, SCHEFFRAHN I, BARTLING S, WEIS J, VON F, V, MIDDLETON M, KATO M, ERGUN S, SCHADENDORF D (2010). Resistance to antiangiogenic therapy is directed by vascular phenotype, vessel stabilization, and maturation in malignant melanoma. J Exp Med 207: 491-503.

HILLEN F, GRIFFIOEN AW (2007). Tumour vascularization: sprouting angiogenesis and beyond. Cancer Metastasis Rev 26: 489-502.

HLUSHCHUK R, BAUM O, GRUBER G, WOOD J, DJONOV V (2007). The synergistic action of a VEGF-receptor tyrosine-kinase inhibitor and a sensitizing PDGF-receptor blocker depends upon the stage of vascular maturation. Microcirculation 14: 813-825.

HLUSHCHUK R, RIESTERER O, BAUM O, WOOD J, GRUBER G, PRUSCHY M, DJONOV V (2008). Tumor recovery by angiogenic switch from sprouting to intussusceptive angiogenesis after treatment with PTK787/ZK222584 or ionizing radiation. Am J Pathol 173: 1173-1185.

JAIN RK (2005). Normalization of tumor vasculature: an emerging concept in antiangiogenic therapy. Science 307: 58-62.

JAIN RK (2008). Lessons from multidisciplinary translational trials on anti-angiogenic therapy of cancer. Nat Rev Cancer 8: 309-316.

JAIN RK, DUDA DG, CLARK JW, LOEFFLER JS (2006). Lessons from phase III clinical trials on anti-VEGF therapy for cancer. Nat Clin Pract Oncol 3: 24-40.

KRAMER I, LIPP HP (2007). Bevacizumab, a humanized anti-angiogenic monoclonal antibody for the treatment of colorectal cancer. J Clin Pharm Ther 32: 1-14.

KU DD, ZALESKI JK, LIU S, BROCK TA (1993). Vascular endothelial growth factor induces EDRF-dependent relaxation in coronary arteries. Am J Physiol 265: H586-H592.

MA J, WAXMAN DJ (2008). Combination of antiangiogenesis with chemotherapy for more effective cancer treatment. Mol Cancer Ther 7: 3670-3684.

MAKANYAAN, HLUSHCHUK R, DJONOV VG (2009). Intussusceptive angiogenesis and its role in vascular morphogenesis, patterning, and remodeling. Angiogenesis 12: 113-123.

MILLER KD, SWEENEY CJ, SLEDGE GW, JR. (2003). The Snark is a Boojum: the continuing problem of drug resistance in the antiangiogenic era. Ann Oncol 14: $20-28$.

MILLER KD, SWEENEY CJ, SLEDGE GW, JR. (2005). Can tumor angiogenesis be inhibited without resistance?. In Mechanisms of Angiogenesis (Eds. M. Clauss, G. Breier). EXS. Birkhäuser, Basel, pp. 95-112.

NAKAMURA K, TAGUCHI E, MIURA T, YAMAMOTO A, TAKAHASHI K, BICHAT F, GUILBAUD N, HASEGAWA K, KUBO K, FUJIWARA Y, SUZUKI R, KUBO K, SHIBUYA M, ISAE T (2006). KRN951, a highly potent inhibitor of vascular endothelial growth factor receptor tyrosine kinases, has antitumor activities and affects functional vascular properties. Cancer Res 66: 9134-9142.

NAKAMURA K, YAMAMOTO A, KAMISHOHARA M, TAKAHASHI K, TAGUCHI E, MIURA T, KUBO K, SHIBUYA M, ISOE T (2004). KRN633: A selective inhibitor of vascular endothelial growth factor receptor-2 tyrosine kinase that suppresses tumor angiogenesis and growth. Mol Cancer Ther 3: 1639-1649.

RUDIN M, MCSHEEHY PM, ALLEGRINI PR, RAUSCH M, BAUMANN D, BECQUET M, BRECHTK, BRUEGGEN J, FERRETTIS, SCHAEFFER F, SCHNELLC, WOOD $\mathrm{J}$ (2005). PTK787/ZK222584, a tyrosine kinase inhibitor of vascular endothelial growth factor receptor, reduces uptake of the contrast agent GdDOTA by murine orthotopic B16/BL6 melanoma tumours and inhibits their growth in vivo. NMR Biomed 18: 308-321

RUGGERI B, SINGH J, GINGRICH D, ANGELES T, ALBOM M, YANG S, CHANG $H$, ROBINSON C, HUNTER K, DOBRZANSKI P, JONES-BOLIN S, PRITCHARD S, AIMONE L, KLEIN-SZANTO A, HERBERT JM, BONO F, SCHAEFFER P, CASELLAS P, BOURIE B, PILI R, ISAACS J, ATOR M, HUDKINS R, VAUGHT J, MALLAMO J, DIONNE C (2003). CEP-7055: a novel, orally active pan inhibitor of vascular endothelial growth factor receptor tyrosine kinases with potent antiangiogenic activity and antitumor efficacy in preclinical models. Cancer Res 63: 5978-5991.

SENNINO B, KUHNERT F, TABRUYN SP, MANCUSO MR, HU-LOWE DD, KUO CJ, MCDONALD DM (2009). Cellular source and amount of vascular endothelia growth factor and platelet-derived growth factor in tumors determine response to angiogenesis inhibitors. Cancer Res 69: 4527-4536.

SOLTAU J, DREVS J (2009). Mode of action and clinical impact of VEGF signaling inhibitors. Expert Rev Anticancer Ther 9: 649-662.

VAN HINSBERGH VW, KOOLWIJKP (2008). Endothelial sprouting and angiogenesis: matrix metalloproteinases in the lead. Cardiovasc Res 78: 203-212.

YOSHIJI H, HARRIS SR, THORGEIRSSON UP (1997). Vascular endothelial growth factor is essential for initial but not continued in vivo growth of human breast carcinoma cells. Cancer Res 57: 3924-3928. 


\section{Further Related Reading, published previously in the Int. J. Dev. Biol.}

The seminal work of Werner Risau in the study of the development of the vascular system Domenico Ribatti

Int. J. Dev. Biol. (2010) 54: 567-572

\section{Embryonic development of the proepicardium and coronary vessels} Anna Ratajska, Elzbieta Czarnowska and Bogdan Ciszek

Int. J. Dev. Biol. (2008) 52: 229-236

Vasculogenesis and angiogenesis in the mouse embryo studied using quail/mouse chimeras Michel Pudliszewski and Luc Pardanaud

Int. J. Dev. Biol. (2005) 49: 355-361

Vascular development: from precursor cells to branched arterial and venous networks

Anne Eichmann, Li Yuan, Delphine Moyon, Ferdinand leNoble, Luc Pardanaud and Christiane Bréant Int. J. Dev. Biol. (2005) 49: 259-267

Parallels in invasion and angiogenesis provide pivotal points for therapeutic intervention Suzanne A. Eccles

Int. J. Dev. Biol. (2004) 48: 583-598

ISI Impact Factor $(\mathbf{2 0 1 0})=2.86$

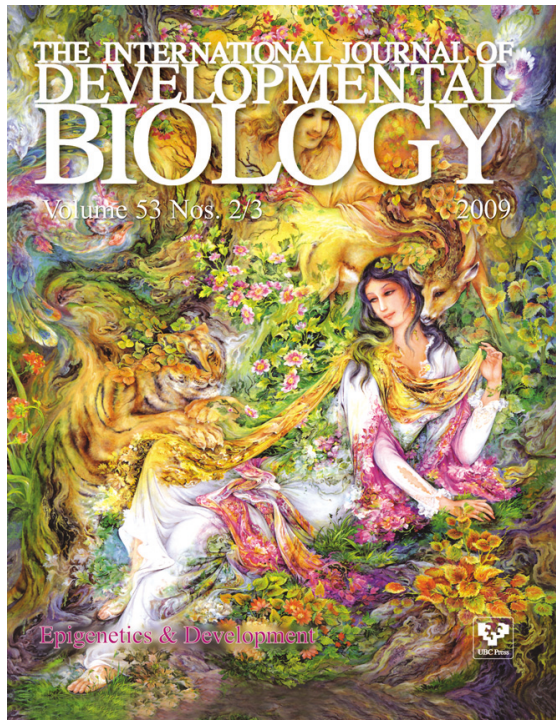

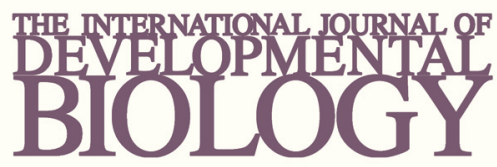

Volume 54 Nos. 6/7
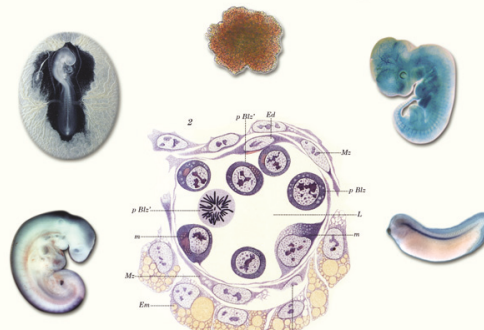

Special Issue
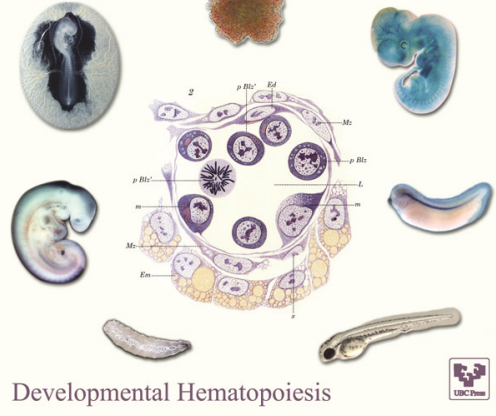

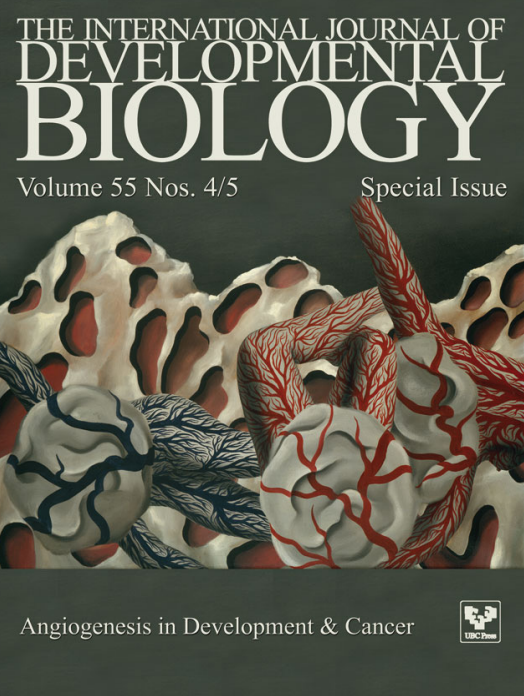

